\title{
Beampattern Synthesis for Frequency Diverse Array Based on Time-Modulated Double Parameters Approach
}

\author{
Tong Mu, Yaoliang Song, Zhonghan Wang \\ School of Electronic and Optical Engineering, Nanjing University of Science and Technology, Nanjing, China. \\ mutong@njust.edu.cn; ylsong@njust.edu.cn; zhwang@njust.edu.cn
}

\begin{abstract}
The basic frequency diverse array (FDA) using linearly increasing frequency increments generates a range-angle dependent beampattern. However, it is coupled in range and angle dimensions and is also periodic in range and time, making its applications limited. In this paper, a novel FDA beampattern synthesis approach is proposed utilizing the time-modulated double parameters based on the chaos sequence. The chirp signal mechanism is used instead of the single-frequency signal mechanism. Meanwhile, the multi-carrier architecture is used for range-angle decoupling. Satisfactory time-invariant range-angle beampattern can be synthesized for both single and multiple targets locations. Simulation results show the effectiveness of the proposed FDA scheme. Furthermore, comparative study with the existing technology indicates that the proposed approach can provide better performance in spatial focusing and side-lobe suppressing.
\end{abstract}

Index Terms - beampattern synthesis, chaos sequence, frequency diverse array, time modulation.

\section{INTRODUCTION}

First proposed in [1] and [2], frequency diverse array (FDA) technology generates beampatterns that depend on the range, angle and time by introducing a tiny frequency offset among the array elements. In a conventional array, the beampattern can be focused to the desired angle utilizing the progressive phase shift (PPS) between the elements [3-4], but the beam steering is fixed in an angle for all range cells. The FDA transmits the beampattern over the desired range and angle thus it can suppress range-dependent interferences and clutters which is desirable in radar applications. Besides, the FDA provides new degrees of freedom in the range, angle and time for designing the array factor, which enables the beam to scan without the need of phase shifters or mechanical steering [5]. Therefore the FDA can offer much capabilities such as synthetic aperture radar imaging [6], auto scanning [7] and localization of targets [8], etc.

In the basic FDA, the linearly increasing frequency increments are applied and result in a beampattern coupled in range and angle domains, which has a ' $S$ ' shape [5]. This characteristic limits FDA's applications in unambiguously localizing targets in range-angle dimension [9]. Moreover, the basic FDA beampattern is periodic in range and time, deteriorating the resulting signal-to- 
interference-plus-noise ratio (SINR) and target detectability. The range and time periodicity of FDA beampattern is analyzed in [10], and the range-angle coupled beamforming has been investigated using frequency-diverse chips in [11]. A logarithmic frequency offset scheme is proposed in [12] which can decouple the beampattern and eliminate the range periodicity. However, its reduced rangeangle resolution and enhanced side-lobes may not be acceptable for most applications. A FDA system based on multi-carrier frequencies with logarithmic frequency offset is proposed in [13], which requires additional convex optimization algorithm for synthesizing the complex element weights to generate dot-shaped beampatterns. Particularly, the beampatterns generated by these approaches are time-variant, causing the loss in SINR and seriously affecting the target detectability because of extremely short duration of the beam illumination at the target position. Time-independent beampattern based on time-modulated frequency offset (TMFO) is proposed in [14], but the range periodicity is still not eliminated. Then, time-invariant beampattern with a single maximum at the target location is achieved in [15] using the time-modulated logarithmically increasing frequency offset (TMLFO). Also in [15], time-modulated optimized frequency offset (TMOFO) is developed for obtaining better spatial focusing performance. Recently, multi-carrier architecture combined with nonlinear distributed frequency offset is proposed in [16] and pencil-shaped time-invariant beampattern is achieved.

The existing research are based on the single-frequency signal mechanism and thus only single parameter modulation is considered, namely the frequency offset modulation. In this paper, a novel FDA beampattern synthesis approach is proposed based on the chirp signal mechanism where frequency offset as well as chirp constant modulation are both considered. The Logistic mapping chaos sequence is applied for the double parameter modulation, and the multi-carrier architecture is employed to decouple the beampattern in range and angle dimensions. Time-invariant beampattern at the desired location can be achieved by the proposed approach. Also, the time window version of the proposed FDA is used for multiple targets scenario. Simulation experiments are carried out to evaluate the performance of the proposed approach. The results are also compared with the MultiCarrier-FDA (MC-FDA) recently proposed in [16] and it turned out that the proposed FDA can provide improved spatial focusing performance with more effective side-lobe suppression simultaneously. 


\section{TIME-MODULATED DOUBLE-PARAMETER FDA}

\section{A. Signal model}

Consider an antenna array of $M$ identical transmit elements with uniform spacing $d$.

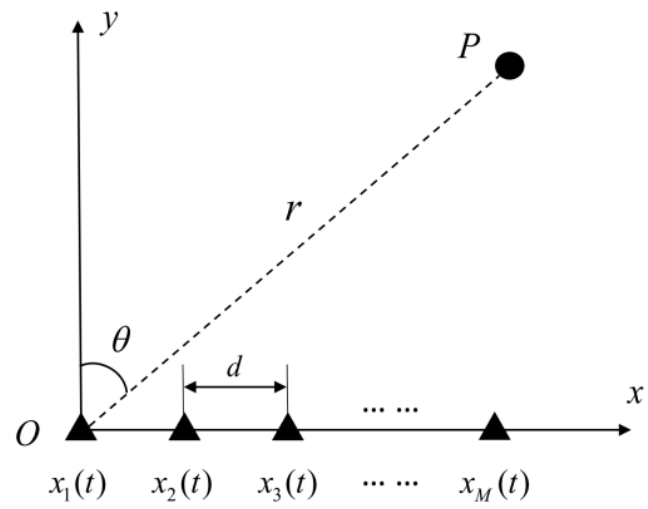

Fig. 1. Diagram of the proposed FDA.

As shown in Fig. 1, $P$ is an observation point in the far field, and $r_{m}$ is the distance from $P$ to the $m$ th element. Multi-carrier framework is considered in the system thus each transmit element emits a weighted summation of a series of basic signals. Unlike the previous research, the chirp signal instead of the single-frequency signal is used here as the carrier. Suppose the number of carriers at each element is $N$, then the signal transmitted from the $m$ th element is

$$
x_{m}(t)=\sum_{n=0}^{N-1} a_{n, m} e^{-j 2 \pi\left(f_{n, m} t+\frac{1}{2} u_{n, m} t^{2}\right)}, 0 \leq t \leq T, 0 \leq m \leq M-1
$$

where $a_{n, m}, f_{n, m}$ and $u_{n, m}$ are the complex weight used for steering the maximum of the beampattern at the target location, radiation frequency and chirp constant for the $n$th carrier at the $m$ th element, respectively. $T$ is the duration of the transmitted pulse signal. The frequency component $f_{n, m}$ can be expressed as

$$
f_{n, m}=f_{0}+\Delta f_{n, m}, 0 \leq n \leq N-1,0 \leq m \leq M-1
$$

where $f_{0}$ is the reference frequency and $\Delta f_{n, m}$ is the frequency offset.

Therefore, the overall signal observed at an arbitrary far field point $P(r, \theta)$ is

$$
X(t, r, \theta)=\sum_{m=0}^{M-1} \sum_{n=0}^{N-1} a_{n, m} e^{-j 2 \pi\left[f_{n, m}\left(t-\frac{r_{m}}{c}\right)+\frac{1}{2} u_{n, m}\left(t-\frac{r_{m}}{c}\right)^{2}\right]}
$$

where $c$ is the speed of light in free space, $r$ and $\theta$ are the range and azimuth angle from the point to the first element respectively, and $r_{m}$ is the distance between the point and the $m$ th element. Taking the far field approximation into consideration yields

$$
r_{m} \approx r-m d \sin \theta
$$

Substituting (2) and (4) into (3) yields 


$$
\begin{aligned}
& X(t, r, \theta)=\sum_{m=0}^{M-1} \sum_{n=0}^{N-1} a_{n, m} e^{-j 2 \pi\left[\left(f_{0}+\Delta f_{n, m}\right)\left(t-\frac{r-m d \sin \theta}{c}\right)+\frac{1}{2} u_{n, m}\left(t-\frac{r-m d \sin \theta}{c}\right)^{2}\right]} \\
& =e^{-j 2 \pi f_{0}\left(t-\frac{r}{c}\right)} \sum_{m=0}^{M-1} \sum_{n=0}^{N-1} a_{n, m} e^{-j 2 \pi\left\{\frac{f_{0} m d \sin \theta}{c}+\Delta f_{n, m}\left(t-\frac{r}{c}\right)+\frac{\Delta f_{n, m} m d \sin \theta}{c}+\frac{1}{2} u_{n, m}\left[\left(t-\frac{r}{c}\right)^{2}+\left(\frac{m d \sin \theta}{c}\right)^{2}+\frac{2\left(t-\frac{r}{c}\right) m d \sin \theta}{c}\right]\right\}}
\end{aligned}
$$

In practical FDA system, the frequency offset satisfies

$$
\max \left\{\left|\Delta f_{n, m}\right|\right\}<<f_{0}
$$

thus the term $\frac{\Delta f_{n, m} m d \sin \theta}{c}$ can be neglected from (5). Besides, the term $\left(\frac{m d \sin \theta}{c}\right)^{2}+\frac{2\left(t-\frac{r}{c}\right) m d \sin \theta}{c}$ in (5) can also be ignored because

$$
\begin{gathered}
\frac{1}{2} u_{n, m}\left(\frac{m d \sin \theta}{c}\right)^{2}<<\frac{\Delta f_{n, m} m d \sin \theta}{c} \\
\frac{1}{2} u_{n, m} \frac{2\left(t-\frac{r}{c}\right) m d \sin \theta}{c}<<\Delta f_{n, m}\left(t-\frac{r}{c}\right)
\end{gathered}
$$

Therefore, (5) can be further approximated as

$$
X(t, r, \theta) \approx e^{-j 2 \pi f_{0}\left(t-\frac{r}{c}\right)} \sum_{m=0}^{M-1} \sum_{n=0}^{N-1} a_{n, m} e^{-j 2 \pi\left[\frac{f_{0} m d \sin \theta}{c}+\Delta f_{n, m}\left(t-\frac{r}{c}\right)+\frac{1}{2} u_{n, m}\left(t-\frac{r}{c}\right)^{2}\right]}
$$

Then the corresponding array factor (AF) can be obtained

$$
A F(t, r, \theta)=\sum_{m=0}^{M-1} \sum_{n=0}^{N-1} a_{n, m} e^{-j 2 \pi\left[\frac{f_{0} m d \sin \theta}{c}+\Delta f_{n, m}\left(t-\frac{r}{c}\right)+\frac{1}{2} u_{n, m}\left(t-\frac{r}{c}\right)^{2}\right]}
$$

\section{B. Beampattern synthesis based on the proposed FDA}

Linear incremental frequency offset makes the FDA beampattern periodic in range and time, while nonlinear distributed time-modulated frequency offset can result in time-invariant spatial focusing beampattern. In order to eliminate the time-variant and range-periodic characteristics of the beampattern, and also improve the performance of the FDA system in spatial focusing and side-lobe suppressing, a new scheme based on the time-dependent frequency offset and chirp constant is designed, namely the Time-Modulated Double-Parameter FDA (TMDP-FDA). Suppose the target position is $\left(r_{0}, \theta_{0}\right)$, and for the $n$th carrier at the $m$ th element, the frequency offset and chirp constant are defined as

$$
\begin{gathered}
\Delta f_{n, m}=\Delta f \frac{p_{n, m}}{t-\frac{r_{0}}{c}} \\
u_{n, m}=\Delta u \frac{2 q_{n, m}}{\left(t-\frac{r_{0}}{c}\right)^{2}}
\end{gathered}
$$


respectively with $t \in[0, T]$ and $T<<\frac{r_{0}}{c} . \Delta f$ and $\Delta u$ are the frequency offset and chirp constant controlling coefficients respectively. For each element, $p_{n, m}$ and $q_{n, m}$ are generated by the chaos sequence based on the Logistic mapping and they are given by

$$
\begin{aligned}
& p_{n, m}=\left\{\begin{array}{l}
p_{0, m}, n=0 \\
4 p_{n-1, m}\left(1-p_{n-1, m}\right), 1 \leq n \leq N-1
\end{array}\right. \\
& q_{n, m}=\left\{\begin{array}{l}
q_{0, m}, n=0 \\
4 q_{n-1, m}\left(1-q_{n-1, m}\right), 1 \leq n \leq N-1
\end{array}\right.
\end{aligned}
$$

where $p_{0, m}$ and $q_{0, m}$ are the initial values for the $m$ th element and satisfy $0<p_{0, m}<1$ and $0<q_{0, m}<1$, respectively. The transmit beampattern can be decoupled as range and angle parts by applying the multi-carrier architecture [16], therefore the resulting array factor can be expressed as

$$
A F(t, r, \theta)=\sum_{m=0}^{M-1} \sum_{n=0}^{N-1} a_{\theta} e^{-j 2 \pi \frac{f_{0} m d \sin \theta}{c}} a_{r} e^{-j 2 \pi\left[\Delta f \frac{p_{n, m}}{\left.t-\frac{r_{0}}{c}\left(t-\frac{r}{c}\right)+\frac{1}{2} \Delta u \frac{2 q_{n, m}}{\left(t-\frac{r_{0}}{c}\right)^{2}}\left(t-\frac{r}{c}\right)^{2}\right]}\right.}
$$

with $a_{n, m}=a_{\theta} a_{r}$. Obviously, the beampattern value of (15) is maximum for $t=0, r=0, \theta=0$. To steer the maximum at the desired target position $\left(r_{0}, \theta_{0}\right)$, the corresponding complex weights are designed as

$$
\begin{gathered}
a_{\theta}=e^{j 2 \pi \frac{f_{0} m d \sin \theta_{0}}{c}} \\
a_{r}=e^{j 2 \pi\left(\Delta f p_{n, m}+\Delta u q_{n, m}\right)}
\end{gathered}
$$

respectively. Without loss of generality, the time variable is set as $t=0$, and by substituting (16) and (17) into (15) the array factor can be further deduced as

$$
\left.A F(r, \theta)=\sum_{m=0}^{M-1} \sum_{n=0}^{N-1} e^{-j 2 \pi \frac{f_{0} m d\left(\sin \theta-\sin \theta_{0}\right)}{c}} e^{-j 2 \pi\left[\Delta f \frac{p_{n, m}\left(r-r_{0}\right)}{r_{0}}+\Delta u\right.} \frac{q_{n, m}\left(r^{2}-r_{0}^{2}\right)}{r_{0}^{2}}\right]
$$

Therefore, the beampattern maximum is generated at the desired location based on the designed complex weights.

\section{Beampattern synthesis for multiple targets}

For multiple targets scenario, the time window version of the proposed FDA can be used to generate multiple maximums at the positions of different targets. Suppose there are $I$ targets in the space located at $\left(r_{i}, \theta_{i}\right)$ respectively, $i=1,2, \ldots, I$. Then the duration of single transmit pulse can be uniformly divided into $I$ time windows, as shown in Fig. 2. 


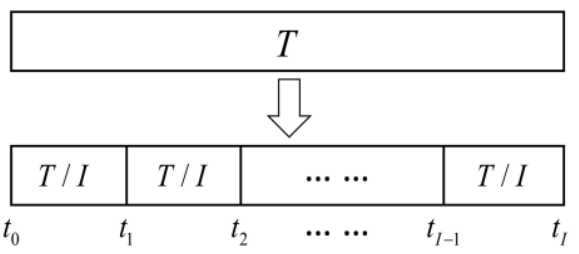

Fig. 2. Division of single pulse duration.

During the $i$ th time window, the frequency offset and chirp constant for the $n$th carrier at the $m$ th element are defined as

$$
\begin{gathered}
\Delta f_{n, m}^{i}=\Delta f \frac{p_{n, m}}{t-\frac{r_{0}(t)}{c}} \\
u_{n, m}^{i}=\Delta u \frac{2 q_{n, m}}{\left(t-\frac{r_{0}(t)}{c}\right)^{2}}
\end{gathered}
$$

respectively where $r_{0}(t)$ is given by

$$
r_{0}(t)=\left[\varepsilon\left(t-t_{i-1}\right)-\varepsilon\left(t-t_{i}\right)\right] r_{i}, t \in\left[t_{i-1}, t_{i}\right)
$$

and

$$
\varepsilon(t)=\left\{\begin{array}{l}
1, t \geq 0 \\
0, t<0
\end{array}\right.
$$

As a result, by combining the time window subdivision with the chirp signal mechanism, the solution for multi-targets beampattern synthesis is given by

$$
A F^{i}(t, r, \theta)=\sum_{m=0}^{M-1} \sum_{n=0}^{N-1} a_{\theta}^{i} e^{-j 2 \pi \frac{f_{0} m d \sin \theta}{c}} a_{r}^{i} e^{-j 2 \pi\left[\Delta f \frac{p_{n, m}}{t-\frac{r_{0}(t)}{c}}\left(t-\frac{r}{c}\right)+\frac{1}{2} \Delta u \frac{2 q_{n, m}}{\left(t-\frac{r_{0}(t)}{c}\right)^{2}}\left(t-\frac{r}{c}\right)^{2}\right]}, t \in\left[t_{i-1}, t_{i}\right)
$$

Similar with (16) and (17), the complex weights for steering the maximum at the $i$ th desired target position $\left(r_{i}, \theta_{i}\right)$ are designed as

$$
\begin{gathered}
a_{\theta}^{i}=e^{j 2 \pi \frac{f_{0} m d \sin \theta_{i}}{c}} \\
a_{r}^{i}=e^{j 2 \pi\left(\Delta f p_{n, m}+\Delta u q_{n, m}\right)}
\end{gathered}
$$

respectively. It can be seen that the angle complex weight needs to be changed for different subpulses, while the range complex weight remains unchanged during the total pulse duration.

\section{NUMERICAL SIMULATIONS}

\section{A. Simulation setup}

In this section, simulation experiments are carried out to examine the effectiveness of the proposed TMDP-FDA. The numerical data are obtained by MATLAB R2013b on the PC with CPU frequency being $3.3 \mathrm{GHz}$ and $\mathrm{RAM}$ being $8 \mathrm{GHz}$, and the processing time for the beampattern synthesis code of the TMDP-FDA is 5.27 seconds. The simulation parameters are set as follows: element number is Brazilian Microwave and Optoelectronics Society-SBMO received 12 Feb 2018; for review 08 Mar 2018; accepted 01 Aug 2018 
$M=15$, carrier number is $N=10$, pulse duration is $T=1 \mathrm{~ms}$ and reference frequency is $f_{0}=5 \mathrm{GHz}$. In order to avoid the grating lobes in angle dimension, the interelement spacing is set as $d=\frac{c}{2 \max \left\{f_{n, m}\right\}}$. The scope of range and angle domains are set as $0 \leq r \leq 1000 \mathrm{~km}$ and $-90^{\circ} \leq \theta \leq 90^{\circ}$ respectively, and the target location is $\left(500 \mathrm{~km}, 30^{\circ}\right)$. The frequency offset and chirp constant controlling coefficients are set as $\Delta f=7$ and $\Delta u=5$ respectively. Two pseudo random sequences each containing $M$ values between 0 and 1 are generated and stored to serve as the initial values of $p_{n, m}$ and $q_{n, m}$.

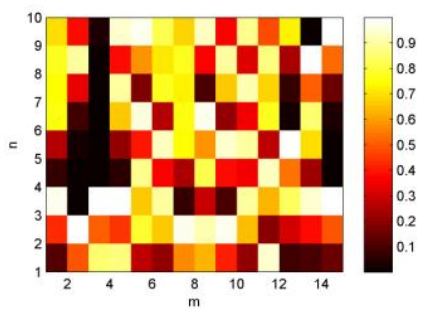

(a)

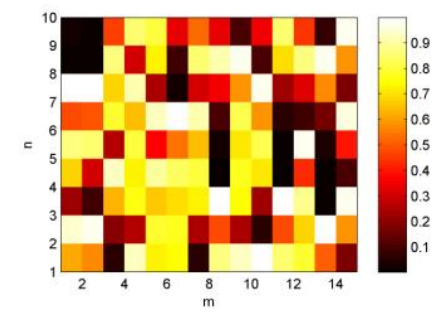

(b)

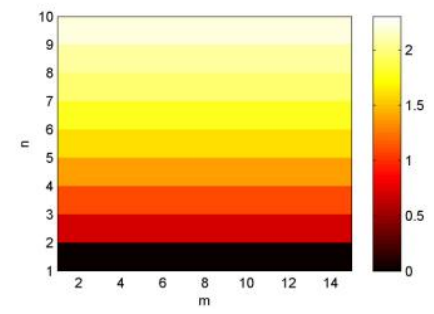

(c)

Fig. 3. Matrix of (a) $p_{n, m}$, (b) $q_{n, m}$ and (c) logarithmic function.

The matrix $\mathbf{P}$ made up of $p_{n, m}$ and $\mathbf{Q}$ made up of $q_{n, m}$ are depicted in Fig. 3 (a) and (b) respectively. The logarithmic function used in MC-FDA for controlling the frequency offset is also shown in Fig. 3 (c) for comparison. It can be seen that for $\mathbf{P}$ and $\mathbf{Q}$, the sequence with respect to each element is nonlinear, non-monotonic, irregular and different from each other. As for Fig. 3 (c), the sequence with respect to each element is nonlinear, monotonic increasing and the same with each other.

\section{B. Results and discussions}

If the time is fixed, the beampattern in a 3-D plot in range and angle domains can be illustrated which contains the spatial information of the beampattern. Fig. 4 shows the normalized beampattern of the proposed TMDP-FDA when $t=0$. From Fig. 4 (a) it can be seen that a single maximum of the beampattern appears right at the desired target location. The range periodicity is eliminated and good range-angle decoupling is realized, indicating the satisfactory spatial focusing performance of the proposed approach. For analyzing the side-lobe level (SLL), the generated beampattern is projected into range and angle dimensions, as shown in Fig. 4 (b) and (c), respectively. It shows that the peak SLL in both range and angle dimensions are below 0.1, indicating the effective side-lobe suppressing performance of the proposed method. The results at $t=0$ obtained by the MC-FDA are depicted in Fig. 5. It shows that the MC-FDA can also produce fine spatial focusing beampattern with low SLL. However, the main-lobe beam width in range dimension of the TMDP-FDA is narrower, which means more energy can be transmitted at the target location and target detecting with higher accuracy can be provided by the proposed FDA. 


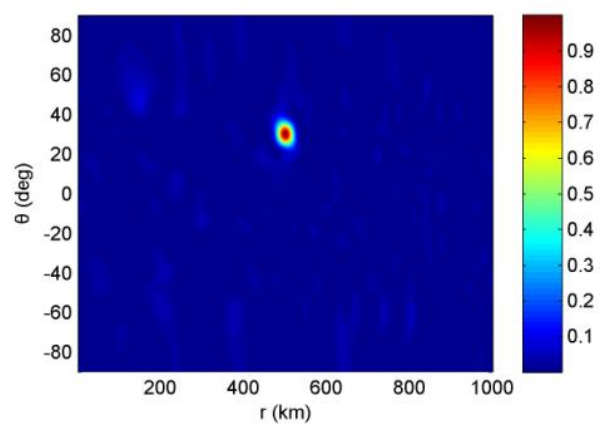

(a)

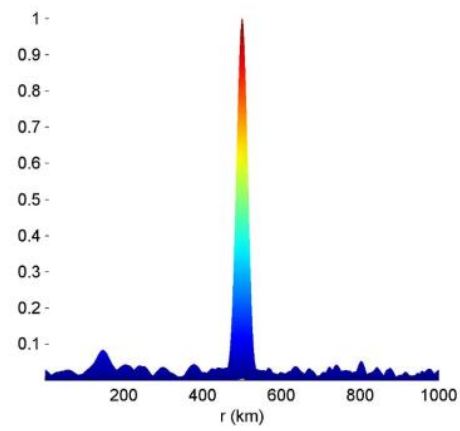

(b)

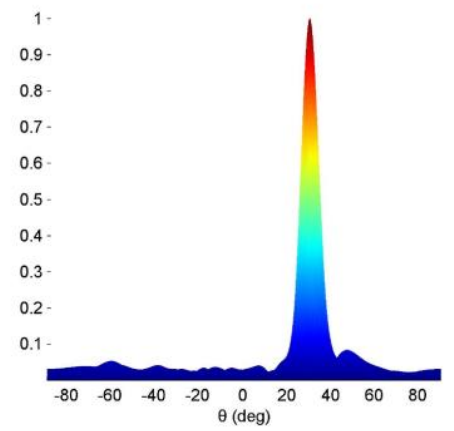

(c)

Fig. 4. Normalized beampattern of the TMDP-FDA in (a) range-angle dimension, (b) range dimension and (c) angle dimension at $t=0$.

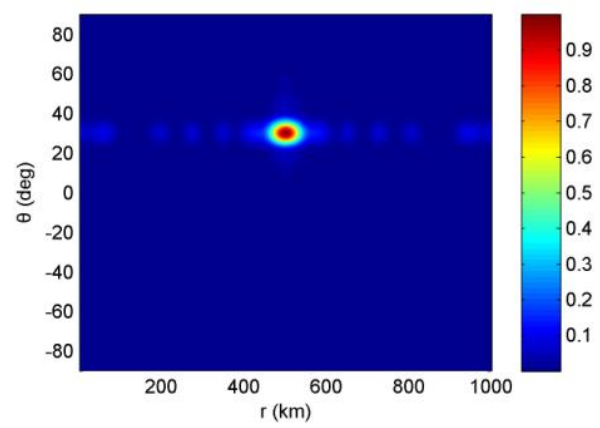

(a)

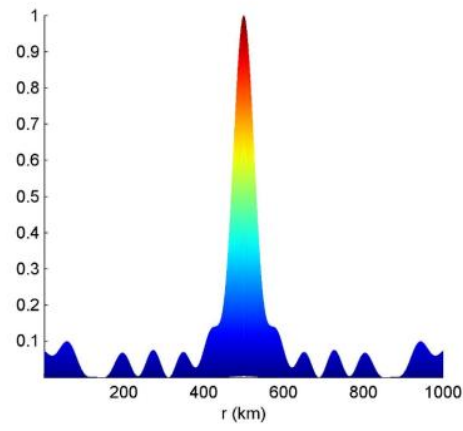

(b)

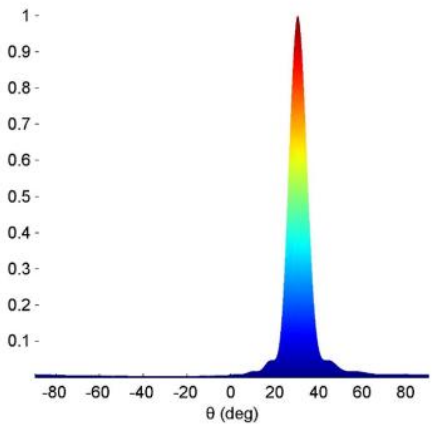

(c)

Fig. 5. Normalized beampattern of the MC-FDA in (a) range-angle dimension, (b) range dimension and (c) angle dimension at $t=0$.

Next the time is fixed at $t=1 \mathrm{~ms}$ and the results of the TMDP-FDA and MC-FDA are shown in Fig. 
Journal of Microwaves, Optoelectronics and Electromagnetic Applications, Vol. 17, No. 3, September 2018 DOI: http://dx.doi.org/10.1590/2179-10742018v17i31240

6 and Fig. 7, respectively. It is observed that the main-lobe beam width in range dimension of the two methods both get narrower, and meanwhile, the peak SLL of the TMDP-FDA is still below 0.1. As for the MC-FDA, several high side-lobes appears in the range dimension and the peak SLL is 0.465.

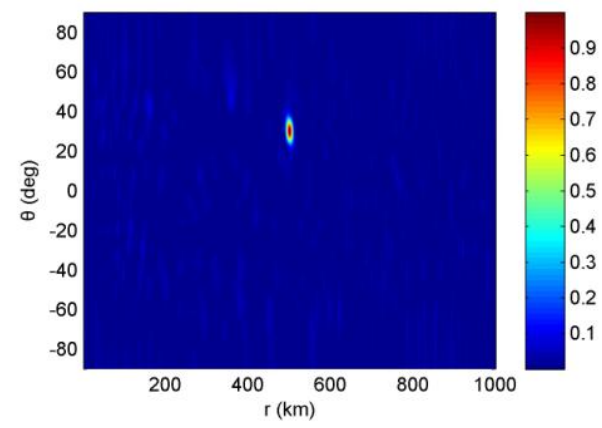

(a)

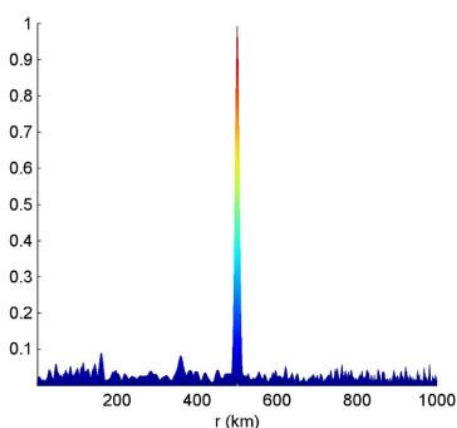

(b)

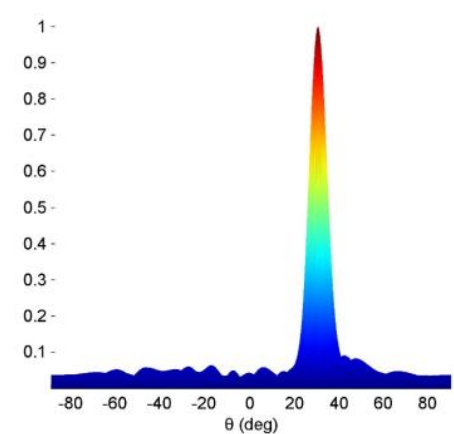

(c)

Fig. 6. Normalized beampattern of the TMDP-FDA in (a) range-angle dimension, (b) range dimension and (c) angle dimension at $t=1 \mathrm{~ms}$.

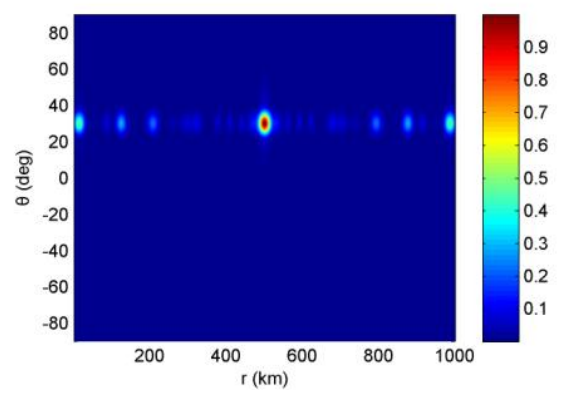

(a)

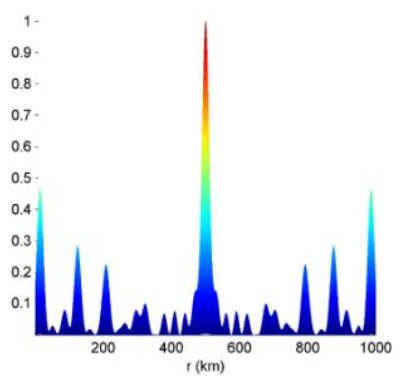

(b)

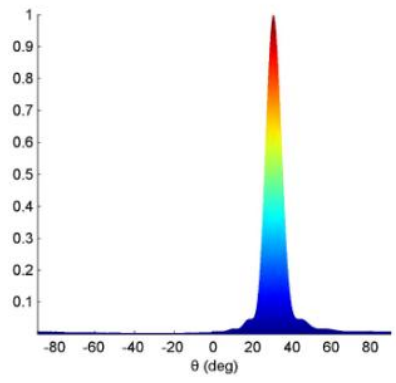

(c)

Fig. 7. Normalized beampattern of the MC-FDA in (a) range-angle dimension, (b) range dimension and (c) angle dimension at $t=1 \mathrm{~ms}$. 
In order to verify the time-invariant characteristic of the TMDP-FDA, the beampattern is projected into time-range and time-angle dimensions, as shown in Fig. 8 (a) and (b), respectively. It is observed that the location of the beampattern maximum in both range and angle dimensions remains unchanged as the time increases in a pulse duration. Correspondingly, Fig. 8 (c) and (d) show the results of the MC-FDA. From Fig. 8 (c) it can be seen that the number and the level of side-lobes in range dimension increase as the time changes, which is not observed in the TMDP-FDA. In addition, the main-lobe beam width in angle dimension of the two methods are the same and do not change with time. It is because the beampattern is totally decoupled as range and angle by applying the multicarrier, and the time modulation acts only on the range dimension. For acquiring narrower beam width in angle dimension, one can increase the number of transmit elements to enlarge the array aperture.

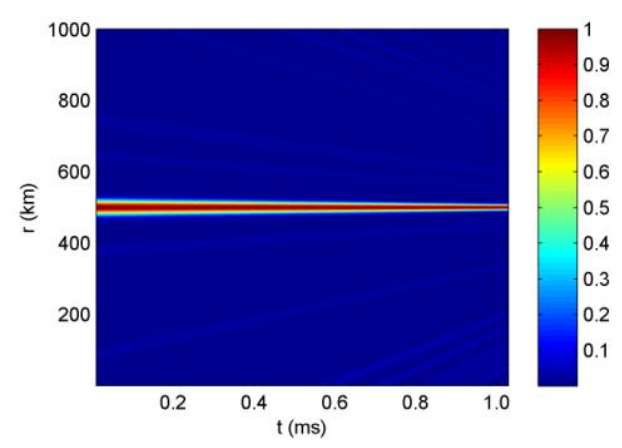

(a)

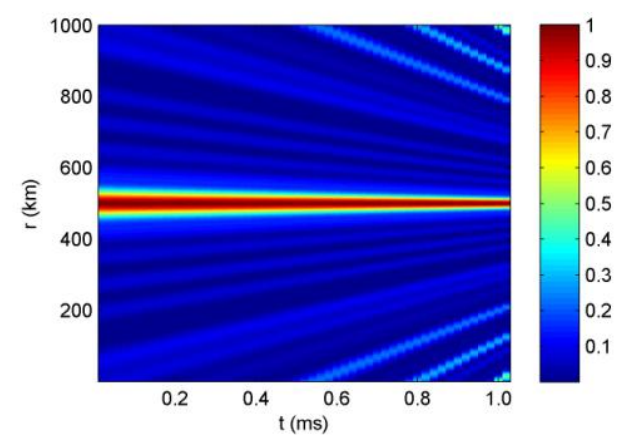

(c)

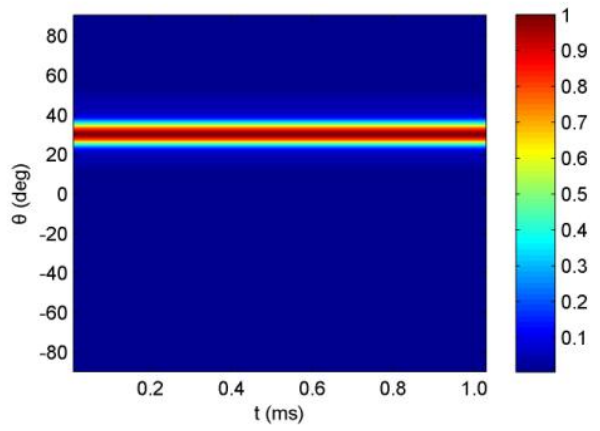

(b)

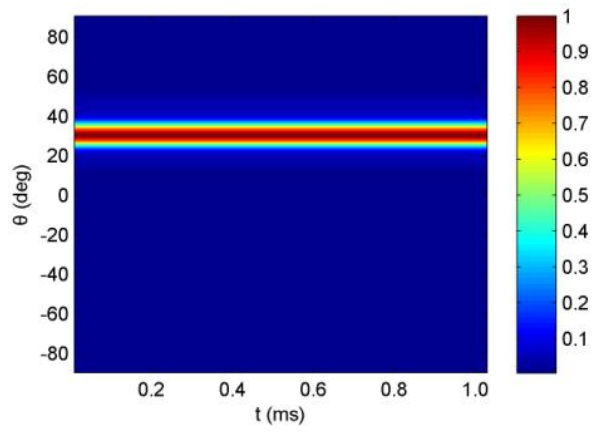

(d)

Fig. 8. Beampattern projection on (a) time-range dimension for TMDP-FDA, (b) time-angle dimension for TMDP-FDA, (c) time-range dimension for MC-FDA and (d) time-angle dimension for MC-FDA.

Comparisons of the main-lobe width in range dimension for three different schemes are provided in Fig. 9, i.e. the TMDP-FDA, the Time-Modulated Single-Parameter FDA (TMSP-FDA) and the MCFDA. Fig. 9 (a) and (b) show the result at $t=0$ and its amplified detail, and Fig. 9 (c) and (d) show the result at $t=1 \mathrm{~ms}$ and its amplified detail. Notice that the only difference between the TMDP-FDA and TMSP-FDA defined here is that the latter uses the single-frequency signal mechanism thus it contains only frequency offset modulation. The results show that the use of chirp signal combined with chaos sequence modulation has better spatial focusing performance than the single-frequency 
signal combined with chaos sequence modulation, both of which are superior to the logarithmic function based single parameter modulation.

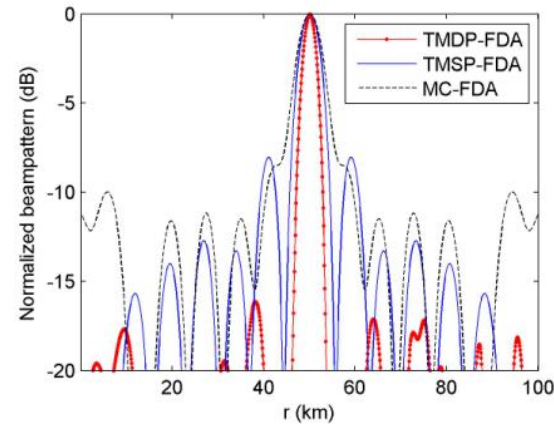

(a)

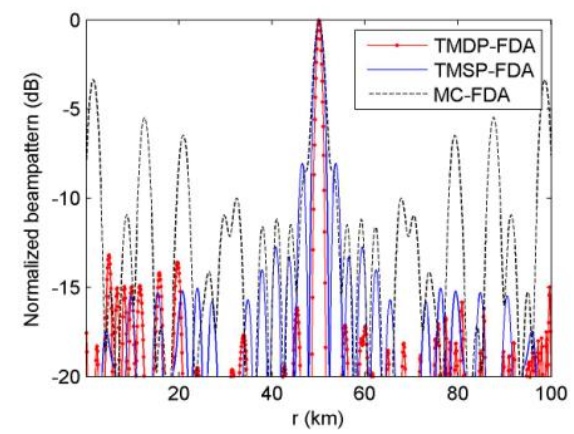

(c)

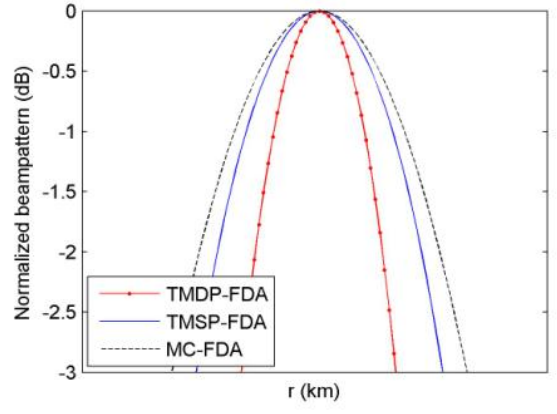

(b)

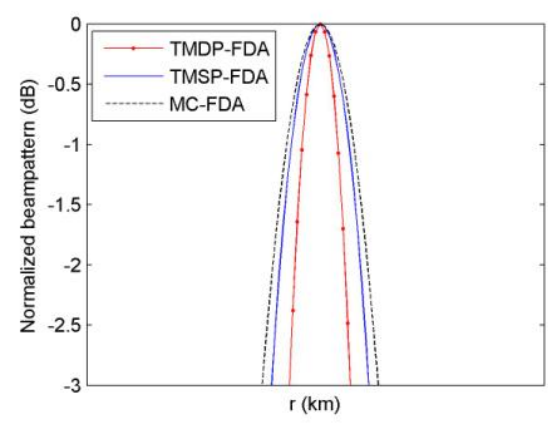

(d)

Fig. 9. Beam width comparisons in range dimension at (a) $t=0$, (b) amplified detail of (a), (c) $t=1 \mathrm{~ms}$ and (d) amplified detail of (c).

The time window version of the proposed TMDP-FDA is used for multiple targets beampattern synthesis. For simplicity, it is supposed that there are two targets located at $\left(450 \mathrm{~km}, 20^{\circ}\right)$ and $\left(700 \mathrm{~km},-30^{\circ}\right)$ respectively. The pulse duration is divided into two time windows, the length of each is $0.5 \mathrm{~ms}$. By fixing the time $t=0$ and $t=0.5 \mathrm{~ms}$ respectively, the resulting normalized beampattern for the two targets is shown in Fig. 10 (a). The beampattern projection on time-range and time-angle dimensions are depicted in Fig. 10 (b) and (c) respectively. It is shown that time-invariant multiple targets beampattern with good spatial focusing can be achieved by the TMDP-FDA as well. 


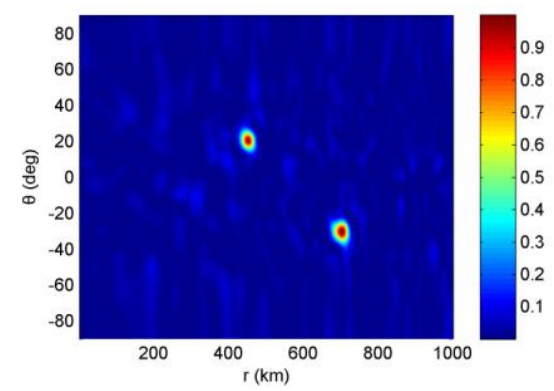

(a)

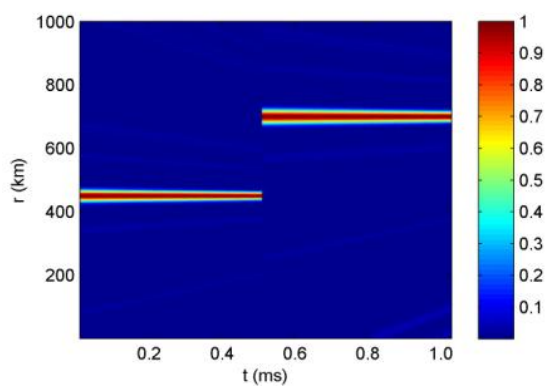

(b)

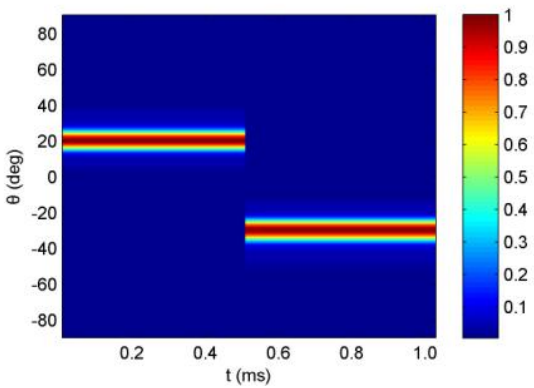

(c)

Fig. 10. (a) Normalized range-angle beampattern for two targets, (b) projection on time-range dimension and (c) projection on time-angle dimension.

Furthermore, the effect of changing the value of frequency offset and chirp constant controlling coefficients to the performance of the TMDP-FDA is investigated. Firstly, the frequency offset is kept as $\Delta f=7$ and $\Delta u$ changes from 1 to 10 and the values of the $-3 \mathrm{~dB}$ main-lobe width in range dimension and the peak SLL for each $\Delta u$ are obtained. Then, the chirp constant controlling coefficient is kept as $\Delta u=5$ and $\Delta f$ changes from 1 to 10 and the corresponding values for each $\Delta f$ are obtained. The results are depicted in Fig. 11. On the one hand, when one of the controlling coefficients is fixed and the other one increases, the $-3 \mathrm{~dB}$ main-lobe width in range dimension decreases, which means narrower beampattern can be obtained. On the other hand, the peak SLL maintains its small value around 0.1 when the coefficient changes. Therefore, based on the TMDPFDA, one can flexibly control the beampattern focusing scope as needed by choosing the appropriate frequency offset and chirp constant controlling coefficients.

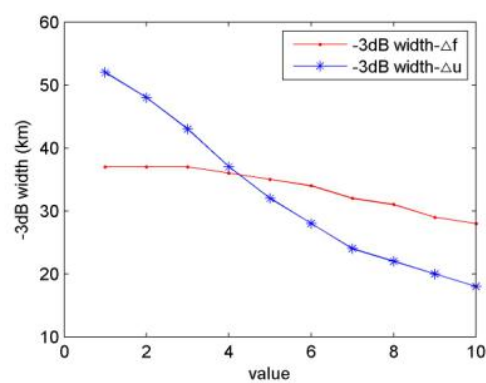

(a)

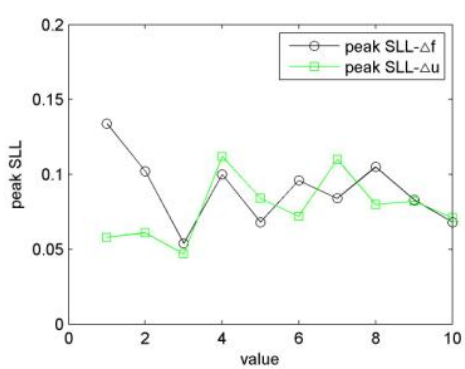

(b)

Fig. 11. (a) Variation curves of the $-3 \mathrm{~dB}$ main-lobe width with respect to $\Delta f$ ( $\Delta u$ ) and (b) variation curves of the peak SLL with respect to $\Delta f(\Delta u)$. 
At last, the initial values $p_{0, m}$ and $q_{0, m}$ are changed by using different pairs of pseudo random sequences, and it turned out that the resulting beampattern is steady in both spatial focusing and SLL. It indicates that the TMDP-FDA is robust to the initial values thus initial value optimization is not necessary, which reduces the system design complexity.

\section{CONCLUSION}

In this paper, a novel TMDP-FDA beampattern synthesis approach has been proposed. Timeinvariant range-angle beampattern is achieved by using the time-modulated double parameters based on the chirp signal mechanism. It is able to generate fine spatial focusing beampattern at the desired location for both single and multiple targets, and it also provides good performance in side-lobe suppressing. Numerical simulations are carried out to evaluate the performance of the proposed TMDP-FDA, and the main results are compared with the MC-FDA. It turned out that at a fixed time the TMDP-FDA has narrower main-lobe beam width in range dimension, and also, it has satisfactorily lower side-lobes with the time changing in a pulse duration. In addition, the beampattern focusing scope of the TMDP-FDA can be flexibly controlled by adjusting the corresponding coefficients and it also reduces the system design complexity.

In future research, the optimal FDA geometric configuration such as the application of planar or distributed geometry and the receive beamforming for the FDA radar need to be investigated. Furthermore, for the practical implementation of the proposed FDA scheme, many issues need to be considered such as generating the waveform, eliminating the intermodulation products of the frequency mixers and reducing the structure complexity, etc [10,17-18].

\section{ACKNOWLEDGMENT}

This work was supported by the National Natural Science Foundation of China under Grant 61271331 and Grant 61571229.

\section{REFERENCES}

[1] P. Antonik, M. C. Wicks, H. D. Griffiths and C. J. Baker, "Frequency diverse array radars," in IEEE Radar Conf., Verona, NY, USA, 2006, pp. 215-217.

[2] M. C. Wicks and P. Antonik, "Frequency diverse array with independent modulation of frequency, amplitude, and phase," U.S. Patent 7319 427, Jan. 15, 2008.

[3] C. A. Balanis, Antenna theory: analysis and design, 3rd ed. New York, NY, USA: John wiley \& sons, 2005.

[4] Y. Konishi, "Phased array antennas," IEICE T. Commun., vol. E86B, no. 3, pp. 954-967, Mar. 2003.

[5] W. Q. Wang, "Frequency diverse array antenna: new opportunities," IEEE Antennas Propag. Mag., vol. 57, no. 2, pp. 145-152, Apr. 2015.

[6] J. Farooq, M. A. Temple and M. A. Saville, "Application of frequency diverse arrays to synthetic aperture radar imaging," in ICEAA, Torino, Italy, 2007, pp. 447-449.

[7] J. J. Huang, K. F. Tong and C. J. Baker, "Frequency diverse array with beam scanning feature," in IEEE Ap-S, San Diego, CA, USA, 2008, pp. 1982-1985.

[8] W. Q. Wang and H. Z. Shao, "Range-angle localization of targets by a double-pulse frequency diverse array radar," IEEE J. Sel. Topics Signal Process., vol. 8, no. 1, pp. 106-114, Feb. 2014.

[9] Y. B. Wang, W. Q. Wang and H. Z. Shao, "Frequency diverse array radar Cramér-Rao lower bounds for estimating direction, range, and velocity," Int. J. Antenn. Propag., vol. 2014, no. 12, pp. 1-15, 2014.

[10] M. Secmen, S. Demir, A. Hizal and T. Eker, "Frequency diverse array antenna with periodic time modulated pattern in range and angle," in IEEE Radar Conf., Boston, MA, USA, 2007, pp. 427-430. 
[11] T. Higgins and S. D. Blunt, "Analysis of range-angle coupled beamforming with frequency-diverse chirps," in $W D D$, Kissimmee, FL, USA, 2009, pp. 140-144.

[12] W. Khan, I. M. Qureshi and S. Saeed, "Frequency diverse array radar with logarithmically increasing frequency offset," IEEE Antennas Wireless Propag. Lett., vol. 14, pp. 499-502, 2015.

[13] H. Z. Shao, J. Dai, J. Xiong, H. Chen and W. Q. Wang, "Dot-shaped range-angle beampattern synthesis for frequency diverse array," IEEE Antennas Wireless Propag. Lett., vol. 15, pp. 1703-1706, 2016.

[14] W. Khan and I. M. Qureshi, "Frequency diverse array radar with time-dependent frequency offset," IEEE Antennas Wireless Propag. Lett., vol. 13, pp. 758-761, 2014.

[15] A. M. Yao, W. Wu and D. G. Fang, "Frequency diverse array antenna using time-modulated optimized frequency offset to obtain time-invariant spatial fine focusing beampattern," IEEE Trans. Antennas Propag., vol. 64, no. 10, pp. 44344446, Oct. 2016.

[16] Y. X. Wang, W. Li, G. C. Huang and J. L. Li, "Time-invariant range-angle-dependent beampattern synthesis for FDA radar targets tracking," IEEE Antennas Wireless Propag. Lett., vol. 16, pp. 2375-2379, 2017.

[17] C. Maffrand, D. Zarate, M. A. Zon, H. Fernandez and M. R. Romero, "Arbitrary waveform generator using FPGA for applications in ultrafast scan voltammetry," in Proc. IEEE IX Southern Conf. Program. Logic, Buenos Aires, Argentina, 2014, pp. 1-6.

[18] T. Eker, S. Demir and A. Hizal, "Exploitation of linear frequency modulated continuous waveform (LFMCW) for frequency diverse arrays," IEEE Trans. Antennas Propag., vol. 61, no. 7, pp. 3546-3553, Jul. 2013. 